Journal of Organometallic Chemistry, 264 (1984) 19-27

Elsevier Sequoia S.A., Lausanne - Printed in The Netherlands

\title{
SYNTHESE UND STRUKTUR DES SÄURE-BASE-ADDUKTES VON METHYLINDIUMDICHLORID UND t-BUTYLAMIN *
}

\author{
M. VEITH* und O. RECKTENWALD \\ Institut für Anorganische und Analytische Chemie der Technischen Universität Braunschweig, \\ Pockelsstr. 4, D-3300 Braunschweig (B.R.D.)
}

(Eingegangen den 8. Juli 1983)

\section{Summary}

Crystals of the Lewis-acid-base adduct $\mathrm{MeInCl}_{2} \cdot \mathrm{H}_{2} \mathrm{~N}^{\mathrm{t}} \mathrm{Bu}$ (I) are formed, when dimethylbis(t-butylamino)silane is allowed to hydrolyze in the presence of methylindium dichloride. I crystallizes in the orthorhombic, acentric space group $P 2_{1} 2_{1} 2_{1}$ with cell dimensions $a$ 1544.6(3), $b$ 1071.4(2), $c$ 628.6(1) pm and $Z=4$. The X-ray structure determination $(R=0.041)$ reveals $I$ to be a polymer in the solid state, the molecules being held together by $\mathrm{N}-\mathrm{H} \cdots \mathrm{Cl}$ interactions, forming an one-dimensional chain structure. The indium atom has a four-fold, tetrahedral coordination. Remarkable intramolecular distances are: N-In 221 pm (very short), In-C(1) 214 pm and $\mathrm{N}-\mathrm{C}(2) 154 \mathrm{pm}$ (both elongated) and In-Cl $240 \mathrm{pm}$. The Lewis acidity of the indium atom in $I$ is compared with that of the tin atom in $\mathrm{Me}_{2} \mathrm{Si}\left(\mathrm{N}^{\mathrm{I}} \mathrm{Bu}\right)_{2} \mathrm{Sn}$. $\mathrm{H}_{2} \mathrm{~N}^{\mathrm{t}} \mathrm{Bu}$ (II) and found to be much higher on the basis of the structural data.

\section{Zusammenfassung}

Kristalle des Lewis-Säure-Base-Adduktes $\mathrm{MeInCl}_{2} \cdot \mathrm{H}_{2} \mathrm{~N}^{t} \mathrm{Bu}$ (I) bilden sich bei der Hydrolyse von Dimethylbis(t-butylamino)silan in Gegenwart von Methylindiumdichlorid. I kristallisiert in der orthorhombischen, acentrischen Raumgruppe $P 2_{1} 2_{1} 2_{1}$ mit den Gitterkonstanten $a$ 1544.6(3), $b$ 1071.4(2), $c$ 628.6(1) pm und mit $Z=4$. Nach der Röntgenstrukturbestimmung $(R=0.041)$ ist $I$ im Festkörper ein Polymer, das über $\mathbf{N}-\mathbf{H} \cdot \cdots \mathrm{Cl}$-Brücken verknüpfte Moleküle enthält. Es liegt eine eindimensionale Kettenstruktur vor. Das Indiumatom ist tetraedrisch von vier Atomen umgeben. Wichtige Bindungslängen im Molekül sind: N-In 221 pm (sehr kurz), In-C(1) $214 \mathrm{pm}$ und N-C(2) $154 \mathrm{pm}$ (beide aufgeweitet) und In-Cl $240 \mathrm{pm}$. Die Lewis-Azidität des Indiumatoms in $I$ ist verglichen mit derjenigen des Zinnatoms in $\mathrm{Me}_{2} \mathrm{Si}\left(\mathrm{N}^{t} \mathrm{Bu}\right)_{2} \mathrm{Sn} \cdot \mathrm{H}_{2} \mathrm{~N}^{t} \mathrm{Bu}$ (II) wesentlich stärker, was sich aus den Strukturdaten ableiten lässt.

\footnotetext{
* Dédié au Doyen Raymond Calas, à l'occasion de son $70^{\circ}$ anniversaire.
} 


\section{Einleitung}

Molekulare Verbindungen des dreiwertigen Indiums InX $X_{3}$ bilden als Lewis-Säuren mit Basen B Verbindungen des Typs $\operatorname{In} X_{3} \cdot \mathbf{B}$ bzw. In $X_{3} \cdot 2 B$. Sind die Substituenten $X$ am Indiumatom elektronenziehend, so werden im allgemeinen zwei Basenmoleküle an einem Indiumatom koordiniert, was zu einer trigonalen Bipyramide um das Zentralatom führt $[1,2]$. Steht für den Substituenten $X$ jedoch ein organischer Rest, so werden $1: 1$-Addukte mit tetraedrisch koordiniertem Indiumatom gefunden [3].

Wir stellen im folgenden die Synthese und Struktur eines Adduktes von Methylindiumdichlorid und t-Butylamin vor, bei dem das Indiumatom trotz zweier elektronenziehender Chlorsubstituenten die Koordinationszahl 4 besitzt. Darüber hinaus interessiert uns diese spezielle Verbindung im Vergleich zu einem kürzlich dargestellten, instabilen Addukt von 1,3-Di-t-butyl-2,2-dimethyl-1,3,2,4 $\lambda^{2}$-diazasilastannetidin mit t-Butylamin (II) [4].

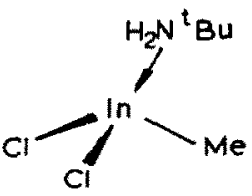

(1)

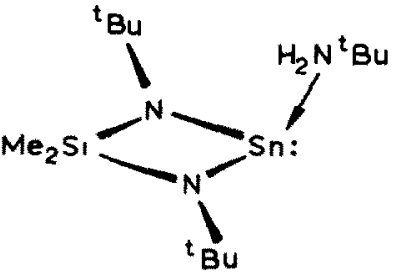

(II)

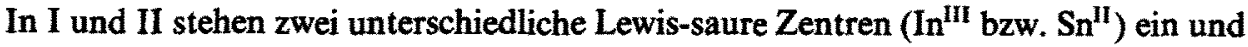
derselben Base (t-Butylamin) gegenüber. Da Grösse und Aussenelektronenzahl der Metallatome sowie die Elektronegativitäten der jeweiligen Substituenten miteinander vergleichbar sind, sollte man aus der Analyse der Strukturdaten interessante Rückschlüsse auf die jeweilige Lewis-Azidität des Indium- und Zinnatoms in I und II ziehen können.

\section{Darstellung des Adduktes von Methylindiumdichlorid mit t-Butylamin (I)}

Lässt man auf eine frisch dargestellte Lösung von Methylindiumdichlorid [5] in Benzol t-Butylamin einwirken, so fällt im Laufe einiger Stunden ein feiner Niederschlag von I aus (G1. 1). Die Mikrokristallinität von I lässt keine Einkristallbeugungsuntersuchungen zu; es ist auch nicht möglich durch Umkristallisieren grössere Spezies zu züchten; da I in Toluol, Dioxan, Diethylether und Acetonitril völlig unlöslich ist. Diese Schwierigkeiten kann man umgehen, wenn man das t-Butylamin erst in einer langsamen Hydrolyse von Dimethylbis(t-butylamino)silan in Anwesenheit von $\mathrm{MeInCl}_{2}$ entstehen lässt (Gl. 2, $\mathrm{Me}=\mathrm{Methyl,}{ }^{\mathrm{t}} \mathrm{Bu}=\mathrm{t}$-Butyl). Unter diesen Bedingungen wachsen neben pulverförmigem Produkt Einkristalle aus der Toluollösung.

$$
\mathrm{Cl}_{2} \mathrm{In}-\mathrm{Me}+{ }^{2} \mathrm{BuNH}_{2} \rightarrow \mathrm{Cl}_{2} \mathrm{In}-\mathrm{Me} \cdot \mathrm{H}_{2} \mathrm{~N}^{\mathrm{t}} \mathrm{Bu}
$$

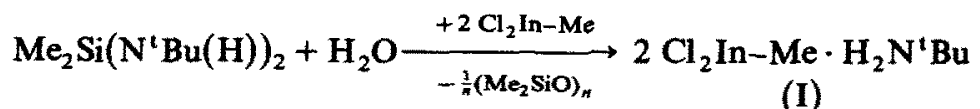


Die Identität des nach beiden Verfahren erhaltenen I wird über vergleichende Pulver-Guinier-Aufnahmen geführt. Die Zusammensetzung von I folgt aus der Analyse. Das gemäss G1. 2 als Nebenprodukt zu erwartende Siloxan kann nicht von weiteren Beiprodukten getrennt werden und wird deshalb auch nicht charakterisiert.

\section{Kristalldaten und Strukturbestimmung von I}

Zur Bestimmung der Raumgruppe eines nadelförmigen Einkristalls von MeIn$\mathrm{Cl}_{2} \cdot \mathrm{H}_{2} \mathrm{~N}^{t} \mathrm{Bu}$ (I) wurden Buerger- und Weissenbergaufnahmen der Schichten $h k 0$ bis $h k 2$ und $h 0 l$ bis $h 3 l$ angefertigt. Die gefundenen Auslöschungen entlang der Gittergeraden führten eindeutig zur acentrischen Raumgruppe $P 22_{1} 2_{1} 2_{1}$. In Tabelle 1 sind die Daten zur Kristall- und Strukturbestimmung von I zusammengefasst. Die Gitterkonstanten wurden an Hand 16 ausgewählter Reflexe im Bereich $25^{\circ} \leq 2 \theta \leq$ $40^{\circ}$ am Diffraktometer bestimmt. Bei der Messung der Reflexintensitäten betrug die Messzeit im Reflexmaximum $20 \mathrm{~s}$ und $10 \mathrm{~s}$ im Untergrund. Zur Lösung der Schweratomstruktur wurden Harkersymmetrien herangezogen. Anschliessende Differenzfouriersynthesen lieferten die Lagen der leichteren Atome $\mathrm{N}$ und $\mathrm{C}$; die Wasserstofflagen konnten nicht ermittelt werden. Die H-Atome der Methylgruppen wurden daher in der gestaffelten Konformation mit einem festen $\mathrm{C}-\mathrm{H}-\mathrm{Abstand}$ von

TABELLE 1

DATEN ZUM KRISTALL UND ZUR STRUKTURBESTIMMUNG VON $\mathrm{MEInCl}_{2} \cdot \mathrm{H}_{2} \mathrm{~N}^{\mathbf{l}} \mathrm{Bu}(\mathrm{I})$

Molmasse 273.9; orthorhombisch, Raumgruppe $P 2{ }_{1} 2{ }_{1} 2_{1} ; a 1544.6(3), b 1071.4(2), c 628.6(1) \mathrm{pm}$, $V 1040.3 \times 10^{6} \mathrm{pm}^{3} ; Z=4 ; D_{\mathrm{x}} 1.75 \mathrm{~g} / \mathrm{m}^{3} ; \mu\left(\mathrm{Mo}-K_{\alpha}\right) 26.4 \mathrm{~cm}^{-1}$, Vierkreisdiffraktometer (Philips, PW 1100 ), Graphitmonochromator, Mo- $K_{\alpha}-$ Strahlung, LP-Korrektur

Kristall: $0.47 \times 0.04 \times 0.03 \mathrm{~mm}^{3} R-$ Wert: $0.041 ; 1714$ Reflexe, davon 127 als "nicht beobachtet" eingestuft;

Strukturbestimmung über Pattersonmethoden

Gewichtsschema: Verfeinerung von $\Sigma\left(|| F_{\mathrm{o}}|-| F_{\mathrm{c}} \mid / W\right)^{2}$ mit $W=1.3077 /\left(\sigma_{(F)}^{2}+0.001014 F^{2}\right)$

TABELLE 2

KOORDINATEN DER ATOME UND ISOTROPE TEMPERATURFAKTOREN ${ }^{a}$ IN DER KRISTALLSTRUKTUR VON I

\begin{tabular}{lllll}
\hline & $x$ & $y$ & $z$ & $\begin{array}{l}B \\
\left(10^{4} \mathrm{pm}^{2}\right)\end{array}$ \\
\hline In & $0.20383(3)$ & $0.22430(4)$ & $0.16380(7)$ & $3.82(3)$ \\
Cl(1) & $0.2032(1)$ & $0.1988(2)$ & $-0.2163(3)$ & $5.7(3)$ \\
Cl(2) & $0.1152(1)$ & $0.0516(2)$ & $0.2680(4)$ & $5.3(3)$ \\
$C(1)$ & $0.1745(6)$ & $0.4076(2)$ & $0.278(2)$ & $6.1(5)$ \\
N & $0.3280(3)$ & $0.1310(5)$ & $0.236(1)$ & $4.1(4)$ \\
H(1) & $0.3183(7)$ & $0.087(2)$ & $0.378(2)$ & $9(1)$ \\
H(2) & $0.3348(8)$ & $0.064(1)$ & $0.120(3)$ & $9(1)$ \\
C(2) & $0.4126(4)$ & $0.2063(7)$ & $0.249(1)$ & $3.9(4)$ \\
C(21) & $0.4215(6)$ & $0.2802(9)$ & $0.049(1)$ & $5.8(5)$ \\
C(22) & $0.4048(7)$ & $0.288(1)$ & $0.442(1)$ & $7.5(6)$ \\
$C(23)$ & $0.4877(5)$ & $0.114(1)$ & $0.272(2)$ & $6.6(5)$ \\
\hline
\end{tabular}

${ }^{a}$ Die angegebenen $B$-Werte des Temperaturfaktors $\exp \left(-B \sin ^{2} \theta / \lambda^{2}\right)$ wurden nach Hamilton [17] aus den $U_{r j}$-Werten von Tab. 3 berechnet. 
TABELLE 3

ANISOTROPE TEMPERATURFAKTOREN $\left(\times 10^{3}\right)^{a}$ DER ATOME VON TABELLE 2

\begin{tabular}{llllllc}
\hline & $U_{11}$ & $U_{22}$ & $U_{33}$ & $U_{23}$ & $U_{13}$ & $U_{12}$ \\
\hline In & $49.1(2)$ & $45.1(2)$ & $51.2(2)$ & $-0.9(2)$ & $-3.1(2)$ & $2.6(2)$ \\
$\mathrm{Cl}(1)$ & $72(1)$ & $91(1)$ & $52.9(8)$ & $-1.3(9)$ & $-8.3(9)$ & $16(1)$ \\
$\mathrm{Cl}(2)$ & $55.2(8)$ & $65(1)$ & $79(1)$ & $13(1)$ & $-10.9(9)$ & $-12.0(8)$ \\
$\mathrm{C}(1)$ & $93(6)$ & $49(4)$ & $90(6)$ & $-1(4)$ & $22(5)$ & $18(4)$ \\
$\mathrm{N}$ & $41(2)$ & $44(3)$ & $70(3)$ & $6(3)$ & $-8(3)$ & $-9(2)$ \\
$\mathrm{C}(2)$ & $50(3)$ & $61(4)$ & $38(3)$ & $6(3)$ & $-9(2)$ & $-18(3)$ \\
$\mathrm{C}(21)$ & $90(5)$ & $76(5)$ & $53(4)$ & $15(4)$ & $0(4)$ & $-35(5)$ \\
$\mathrm{C}(22)$ & $109(7)$ & $126(10)$ & $50(4)$ & $-22(6)$ & $3(5)$ & $-49(7)$ \\
$\mathrm{C}(23)$ & $42(3)$ & $91(6)$ & $119(8)$ & $23(7)$ & $-12(5)$ & $-2(4)$ \\
\hline
\end{tabular}

a Temperaturfaktor: $\exp \left(-2 \pi^{2}\left(U_{11} h^{2} a^{\star 2}+U_{22} k^{2} b^{\star 2}+U_{33} l^{2} c^{\star 2}+2 U_{23} k l a^{\star} c^{\star}+2 U_{13} h l a^{\star} c^{\star}+\right.\right.$ $\left.2 U_{12} h k a^{\star} b^{\star}\right)$ ).

$108 \mathrm{pm}$ und mit Tetraederwinkeln eingegeben, während die H-Atome an dem Stickstoffatom mit einem Abstand N-H 102 pm und einem $\mathrm{H}-\mathrm{N}-\mathrm{H}-$ Winkel von $109^{\circ}$ festgehalten wurden. Der Gütefaktor konvergierte zu $R=0.041$ (siehe auch Tabelle 1). Die Ergebnisse der Strukturbestimmung sind in den Tabellen 2 und 3 aufgelistet. Zur Berechnung der Strukturfaktoren $\left(F_{\mathrm{c}}\right)[6]$ wurden die Atomformfaktoren des SHELX-Systems [7] benutzt.

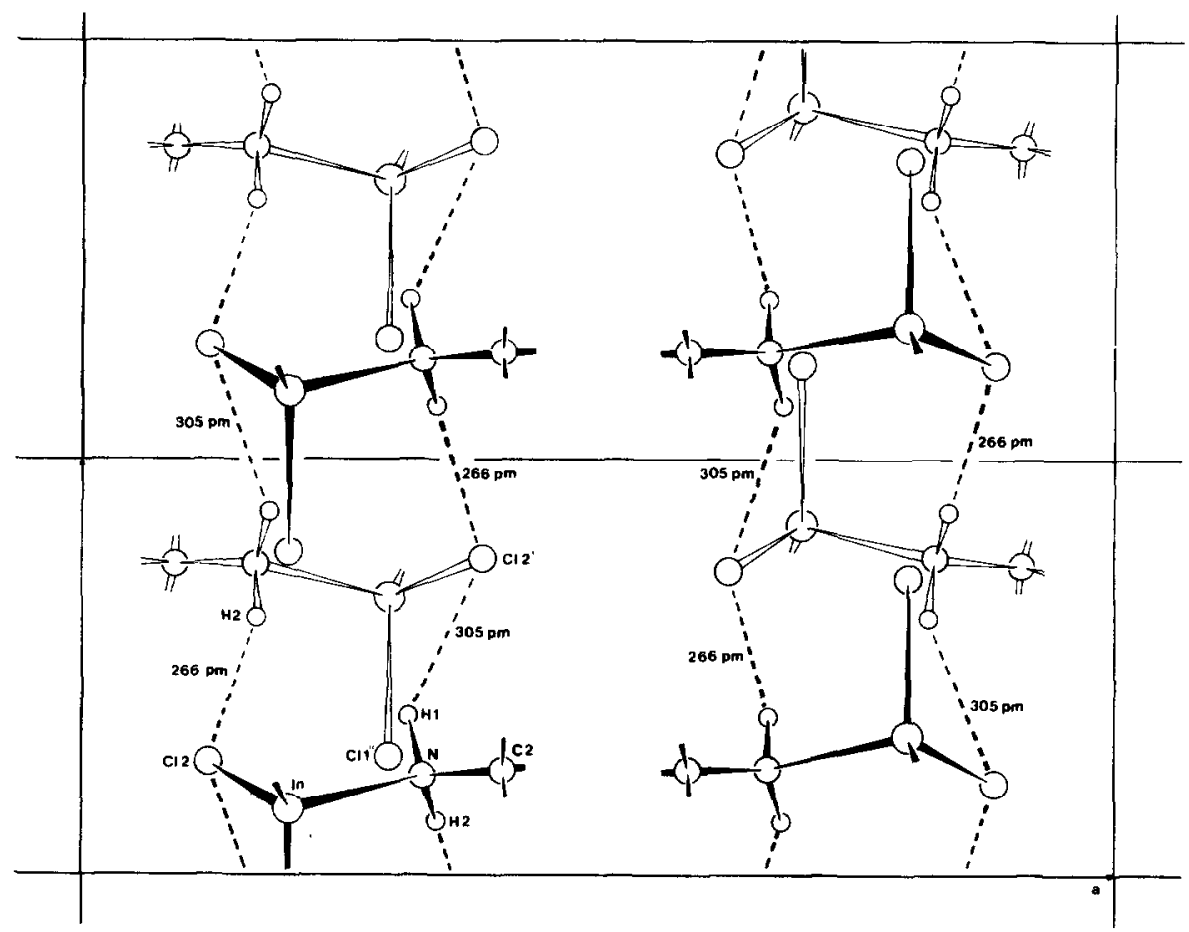

Fig. 1. Die Anordnung der Moleküle I im Kristall (Blickrichtung [010]). Die Methylgruppen sind der besseren Übersicht wegen nicht abgebildet. Die mit gleichartigen Bindungen gezeichneten Moleküle unterscheiden sich in ihrer Höhe um $b / 2$. 


\section{Zur Anordnung der $\mathrm{MeInCl}_{2} \cdot \mathrm{H}_{2} \mathrm{~N}^{\prime} \mathrm{Bu}$-Einheiten im Kristall}

In der Elementarzelle von I befinden sich vier Addukt-Moleküle. Die unabhängig voneinander existierenden Verbindungen $\mathrm{MeInCl}_{2}$ [5] und ' $\mathrm{BuNH}_{2}$ sind in $\mathrm{I}$ über eine In-N-Bindung zu einem Lewis-Säure-Base-Addukt verbunden. Zwischen den Molekülen treten Stickstoff-Wasserstoff-Chlor-Brücken auf, wovon einige in Fig. 1 herausgezeichnet sind. Legt man als Massstab für bindende Wechselwirkungen die Summe der Van-der-Waals-Radien zugrunde $\left(r_{\mathrm{H}}+r_{\mathrm{Cl}}=120+180=300 \mathrm{pm}\right.$ [8] $)$, so findet man besonders kurze Kontakte zwischen $\mathrm{H}\left(2^{\prime \prime}\right)$ und $\mathrm{Cl}(2)$, während alle anderen knapp über diesem Wert liegen: $\mathrm{H}(1) \cdots \mathrm{Cl}\left(2^{\prime \prime}\right) 305, \mathrm{Cl}\left(1^{\prime \prime}\right) \cdots \mathrm{H}(1) 313$ und $\mathrm{Cl}\left(1^{\prime \prime}\right) \cdots \mathrm{H}(2) 306 \mathrm{pm}$.

Man erkennt aus Fig. 1, dass in der Kristallstruktur von I sich Molekülketten ausbilden, die in [001] Richtung (Nadelachse des Kristalls) verlaufen. Zwischen den Ketten bestehen ausnahmslos Van-der-Waals-Kontakte, wobei die kürzesten zwischen Methylgruppen auftreten (H ‥ H 242 pm).

\section{Zur Bindungssituation im Addaktmolekuil I}

Figur 2 veranschaulicht die Anordnung der Atome im Molekül $\mathrm{MeInCl}_{2}$. $\mathrm{H}_{2} \mathrm{~N}^{t} \mathrm{Bu}$, wobei die Wasserstoffatome der Methylgruppen nicht gezeichnet sind. Der zickzackförmige Verlauf der Verbindungslinien zwischen den Atomen $\mathrm{Cl}(2)$, In, $\mathrm{N}$, $C(2)$ und $C(23)$ erinnert stark an die Kettenstruktur gesättigter Kohlenwasserstoffe. Dies ist nicht weiter verwunderlich, da in I ein zum 2,2-Dichlor-4,4-dimethylpentan "Isosteres" vorliegt (bei echter Isosterie sollte für das Indium- ein Boratom stehen). An den Positionen 2 und 3 der Pentankette ist ein Kohlenstoffatom durch ein Element der 3. und der 5. Hauptgruppe ausgetauscht. Wie jedoch die schlechte Löslichkeit der Verbindung zeigt, die offenbar auf die Bildung von Stickstoff-Wasserstoff-Chlor-Brücken zurückzuführen ist (siehe oben), ist die Ähnlichkeit des vorliegenden Moleküls I mit einem gesättigten, substituierten Kohlenwasserstoff nur rein formal gegeben.

Das Molekül I ist im Kristall symmetrielos. Die Abweichung von der höheren Punktsymmetrie $C_{S}(\mathrm{~m})$, die durch ekliptische Stelhung der Methylgruppe $\mathrm{C}(1)$ gegenüber der t-Butylgruppe $\mathbf{C}(2)$ bei Drehung um die In-N-Bindung erreichbar wäre (vergl. Fig. 2), lässt sich mit dem Diederwinkel $\mathrm{C}(1)-\mathrm{In}-\mathrm{N}-\mathrm{C}(2)$ beschreiben, der $35.9^{\circ}$ beträgt. Wegen der intermolekularen Kontakte zwischen den Einheiten I

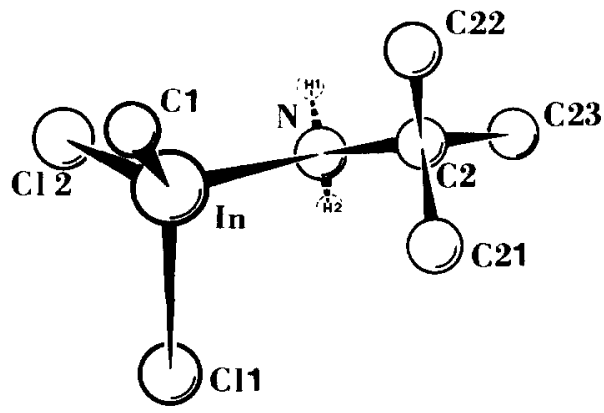

Fig. 2. Die Molekülstruktur von $\mathrm{MeInCl}_{2} \cdot \mathrm{H}_{2} \mathbf{N}^{t} \mathrm{Bu}$ (I) mit [010] als Blickrichtung. 
kristallisiert ein "festgehaltenes" Konformeres aus, das chiral ist. Bemerkenswert an der Struktur von I ist nun, dass auf Grund der Symmetrieoperationen in der Raumgruppe $P 2,2,2$, von den beiden möglichen Rotameren (Atropisomeren) A und B nur das Isomere A vorkommt. Wir haben hier den seltenen Fall vorliegen, dass im

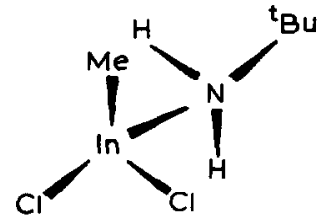

(A)

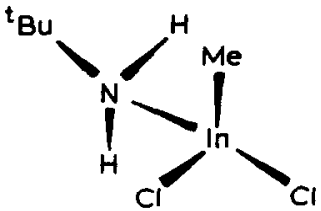

(B)

Kristall nur identische Enantiomere auskristallisieren (Vorraussetzung zur Darstellung optisch reiner Verbindungen). Es ist zu erwarten, dass im Konglomerat auch Kristalle vorkommen, die gerade das andere Enantiomere B enthalten. Im Gegensatz zum Kristall sollten Lösungen von I wegen der zu erwartenden freien Drehbarkeit um die In-N-Bindung die Chiralität verlieren und optisch inaktiv sein.

Das Indiumatom in I ist von vier Atomen verzerrt tetraedrisch umgeben, wie aus den Abständen und Winkeln von Tab. 4 und aus Fig. 3 folgt. Es fällt auf, dass die Atome $\mathrm{N}, \mathrm{Cl}(1)$ und $\mathrm{Cl}(2)$ relativ nahe zusammenrücken, während der Methylgruppe mit dem Zentralatom C(1) ein grosser Raum zukommt (vergl. Winkel am Indiumatom in Tab. 4). Diese Verzerrung von der Tetraedersymmetrie kann man einmal auf die relativ kürzeste In-C(1)-Bindung zurückführen bzw. mit der elektrostatischen Anziehung der mit unterschiedlichen "Partialladungen" behafteten Stickstoff- und Chloratome erklären.

Vergleicht man die Indium-Chlorbindungen im Addukt I mit denjenigen im Methylindiumdichlorid [9], bei dem dem Indiumatom durch intermolekulare Chlor... Indium-Bindungen eine fünffache Koordination zukommt, so sind sie gleich lang (zumindestens, was die kürzesten Abstände angeht), während sie im Dimethylindiumchlorid, das ionische Bindungsanteile besitzt, mit 267 bzw. 295 pm deutlich länger ausfallen [10]. Bei der Adduktbildung zwischen Methylindiumdichlorid und t-Butylamin wird der In-C-Abstand verglichen mit $\mathrm{MeInCl}_{2}$ [9] um 9 pm grösser.

\section{TABELLE 4}

BINDUNGSABSTÄNDE (pm) UND WINKEL $\left({ }^{\circ}\right)$ IM MOLEKÜL I I ${ }^{a}$

\begin{tabular}{lllr}
\hline $\mathrm{In}-\mathrm{Cl}(1)$ & $240.5(2)$ & $\mathrm{Cl}(1)-\mathrm{In}-\mathrm{Cl}(2)$ & $100.5(1)$ \\
$\mathrm{In}-\mathrm{Cl}(2)$ & $239.3(2)$ & $\mathrm{Cl}(1)-\mathrm{In}-\mathrm{C}(1)$ & $115.9(3)$ \\
$\mathrm{In}-\mathrm{C}(1)$ & $213.9(8)$ & $\mathrm{Cl}(1)-\mathrm{In}-\mathrm{N}$ & $98.9(2)$ \\
$\mathrm{In}-\mathrm{N}$ & $220.9(5)$ & $\mathrm{Cl}(2)-\mathrm{In}-\mathrm{C}(1)$ & $119.8(3)$ \\
$\mathrm{N}-\mathrm{C}(2)$ & $153.8(7)$ & $\mathrm{Cl}(2)-\mathrm{In}-\mathrm{N}$ & $95.2(1)$ \\
& & $\mathrm{C}(1)-\mathrm{In}-\mathrm{N}$ & $122.1(3)$ \\
& & $\mathrm{In} \mathrm{N} \mathrm{C}(2)$ & $120.8(4)$
\end{tabular}

Mittelwerte:

t-Butylgr.:

C-C

151(1)

$\mathrm{N}-\mathrm{C}(2)-\mathrm{C}(21.22 .23)$

$107.5(5)$

${ }^{a}$ Bei den Standardabweichungen in Klammern wurden die Ortskoordinatenungenauigkeiten berücksichtigt. 
Zieht man weitere Indiummethylverbindungen zum Vergleich mit hinzu, so findet man z.B. gegenüber dem tetrameren Indiumtrimethyl (kürzester In-C: 206 pm) ebenfalls eine Aufweitung [11]. Auch die Bindung N-C(2) ist mit 154 pm verglichen

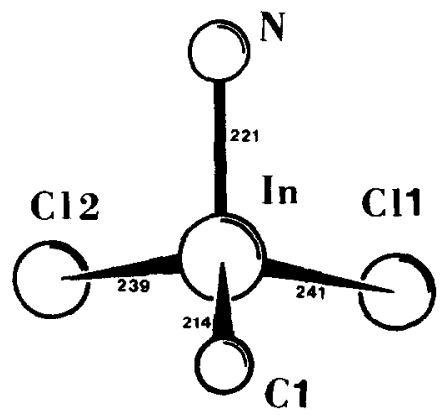

Fig. 3. Die Koordinationsfigur des Indiumatoms in 1 . An $\mathrm{N}$ und $\mathrm{C}(1)$ gebundene Atome sind weggelassen.

mit anderen Verbindungen wie $\left(\mathrm{Sn}\left(\mathrm{N}^{\mathrm{t} B u}\right)_{2} \mathrm{SiMe}_{2}\right)_{2}[12] \operatorname{oder} \mathrm{Sn}_{4}\left(\mathrm{~N}^{\mathrm{t} B u}\right)_{3}\left(\mathrm{OAlMe}_{3}\right)$ [13], in denen die Abstände zwischen dem Zentralatom der t-Butylgruppe und dem $s p^{3}$-hybridisierten, vierfach koordinierten Stickstoffatom im Mittel 146 bzw. 150 pm betragen, besonders gross. Im Addukt von ${ }^{\mathrm{t}} \mathrm{BuNH}_{2}$ mit $\mathrm{Me}_{2} \mathrm{Si}\left(\mathrm{N}^{\prime} \mathrm{Bu}\right)_{2} \mathrm{Sn}$ (II) beträgt der entsprechende N-C-Abstand $147 \mathrm{pm} \mathrm{[4],} \mathrm{ist} \mathrm{also} \mathrm{auch} \mathrm{hier} \mathrm{deutlich}$ kürzer als in $I$.

Wie klar erkennbar ist, erfolgt die Vereinigung der Moleküle ${ }^{\mathrm{t}} \mathrm{BuNH}_{2}$ und $\mathrm{MeInCl}_{2}$ zu $\mathrm{I}$ auf Kosten von bestimmten Bindungselektronendichten in den Ausgangsmolekülen. Die eben herausgestellte besonders lange $\mathrm{N}-\mathrm{C}$-Bindungslänge lässt vermuten, dass es sich bei I um ein aussergewöhnlich stabiles Addukt zwischen der Lewis-Säure $\mathrm{MeInCl}_{2}$ und der Lewis-Base ' $\mathrm{BuNH}_{2}$ handelt. Dies wird eindeutig durch den gefundenen In-N-Abstand bestätigt, der mit 221 pm sehr kurz erscheint. Im $\left(\mathrm{Me}_{2} \operatorname{InNMe}\right)_{2}$, das einen Vierring aus zwei Stickstoff- und zwei Indiumatomen enthält, beträgt die In-N-Bindungslänge $225 \mathrm{pm}$ [14]. In I kommt sicherlich den elektronegativen Chloratomen am Indium sowie auch dem sterisch wenig anspruchsvollen primären Amin eine begünstigende Wirkung auf die Annäherung des Indium- und des Stickstoffatoms zu.

Während die intermolekularen Chlor-Wasserstoffkontakte teilweise im bindenden Bereich liegen, sind die intramolekularen deutlich länger als die Summe der Van-der-Waals-Radien [8]. Der kürzeste Abstand besteht zwischen den Atomen $\mathrm{Cl}(1)$ und $\mathrm{H}(1)$ und beträgt $323 \mathrm{pm}$.

\section{Vergleich zwischen t-Butylamin-Addukten von $\mathrm{Sn}^{\mathrm{II}}$ - und $\mathrm{In}^{\mathrm{III}}-$ Verbindungen}

Molekulare Zinn(II)-Verbindungen wie $\mathrm{Me}_{2} \mathrm{Si}\left(\mathrm{N}^{\prime} \mathrm{Bu}\right)_{2} \mathrm{Sn}[4,15]$ und Indium(III)Verbindungen wie $\mathrm{MeInCl}_{2}$ sind Lewis-Säuren mit einem formalen Elektronensextett. Durch Koordination der Base t-Butylamin erreichen beide Zentralatome Sn und In ein Elektronenoktett (Bildung von II und I). Während I eine stabile Verbindung darstellt mit einem Schmelzpunkt von $117^{\circ} \mathrm{C}$, ist II instabil und zerfällt oberhalb $-40^{\circ} \mathrm{C}$ [4]. Chemisch gesehen ist $\mathrm{MeInCl}_{2}$ eine wesentlich stärkere Lewis-Säure als $\mathrm{Me}_{2} \mathrm{Si}\left(\mathrm{N}^{\mathrm{t}} \mathrm{Bu}\right)_{2} \mathrm{Sn}$. Diese Tatsache spiegelt sich in den Strukturen der Addukte 
wieder. In I steht einem kurzen In-N-Abstand von 221 pm ein langer N-C-Abstand von $154 \mathrm{pm}$ gegenüber. In II ist die Sachlage gerade umgekehrt: die Sn-N-Bindungslänge ist ungewöhnlich lang, während die N-C-Länge normal ausfällt. Die Stabilität des Adduktes korreliert erwartungsgemäss mit dem Element-Stickstoffabstand. Man kann diesen Sachverhalt auch quantitativ fassen: während in I die In-N-Bindungslänge gerade die aus Kovalenzradien berechnete In-N-Standardlänge erreicht (starker Elektronentransfer in die In-N-Bindung), liegt sie bei II um etwa 13\% über der entsprechenden Sn-N-Standardlänge (schwacher Elektronentransfer) [8].

Aus einer anderen Sicht betrachtet bedeuten diese Ergebnisse, dass das Elektronensextett am Zinnatom in $\mathrm{Me}_{2} \mathrm{Si}\left(\mathrm{N}^{t} \mathrm{Bu}\right)_{2} \mathrm{Sn}$ deutlich stabiler ist als dasjenige des Indiumatoms in $\mathrm{MeInCl}_{2}$. Dies spiegelt sich wieder in den Kristallstrukturen der beiden Verbindungen [12,9].

\section{Experimenteller Teil}

I wird in Stock'schen Vakuumapparaturen unter $\mathrm{N}_{2}$ dargestellt. Die $\mathrm{C}, \mathrm{H}$, N-Analysen wurden von der Firma Beller, Göttingen, durchgeführt.

\section{2,2-Dimethyl-4,4-dichlor-3-azonia-4 $\lambda^{4}$-indapentan (I)}

(a) $48 \mathrm{mg}(0.24 \mathrm{mMol})$ Methylindiumdichlorid [5] werden in $5 \mathrm{ml}$ Toluol gelöst und mit $0.24 \mathrm{mMol}$ t-Butylamin versetzt. Nach zweistündigem Rühren wird der farblose, sehr feinkristalline Niederschlag abfiltriert. Eine Guinier-Pulveraufnahme (Hueber, Rimsting) des Produktes ist mit einer entsprechenden Aufnahme des nach (b) gewonnenen Produktes identisch.

(b) $48 \mathrm{mg}(0.24 \mathrm{mMol})$ Methylindiumdichlorid [5] werden in $5 \mathrm{ml}$ Toluol gelöst und mit $30 \mathrm{mg}(0.15 \mathrm{mMol})$ Dimethylbis(t-butylamino)silan [16] versetzt. Nach Zufügen eines Tropfens Wasser bildet sich ein farbloser feiner Niederschlag, auf dem innerhalb mehrer Stunden nadelförmige Kristalle wachsen. Nach $24 \mathrm{~h}$ wird die überstehende Lösung abpipettiert, die Kristalle im Vakuum getrocknet und sortiert. Ausbeute an I: $43.8 \mathrm{mg}$ (67\%). Anal. von I: Gef.: C, $22.17 ; \mathrm{H}, 5.21 ; \mathrm{N}, 5.01$. $\mathrm{C}_{5} \mathrm{H}_{14} \mathrm{NCl}_{2} \mathrm{In}(273.9)$ ber.: $\mathrm{C}, 21.93 ; \mathrm{H}, 5.15 ; \mathrm{N}, 5.11$. Fp. von I: $117^{\circ} \mathrm{C}$.

\section{Dank}

Diese Arbeit wurde mit Mitteln des Landes Niedersachsen gefördert. Wir danken Herrn Prof. U. Thewalt, Ulm, für die Messung der Reflexintensitäten.

\section{Literatur}

1 D.G. Tuck, Coord. Chem. Rev., 1 (1966) 286.

2 M.V. Veidis und G.J. Pelenik, Chem. Commun., (1969) 586.

3 F.G.A. Stone, Chem. Rev., 58 (1958) 101.

4 M. Veith, G. Schlemmer und M.L. Sommer, Z. Anorg. Allg. Chem., 497 (1983) 157.

5 H.C. Clark und A.L. Pickard, J. Organomet. Chem., 8 (1967) 427.

6 O. Recktenwald, Dissertation, Braunschweig 1982. Ein tabell. Vergleich der $F_{0}$ und $F_{\mathrm{e}}$-Werte ist in dieser Arbeit enthalten.

7 G.M. Sheldrick, Program for Crystal Structure Determination, Version SHELX 76.

8 J.E. Huheey, Inorganic Chemistry: Principles of Structure and Reactivity, Harper \& Row, New York 1972, S. 184. 
9 K. Mertz, W. Schwarz, F. Zettler und H.D. Hausen, Z. Naturforsch. B, 30 (1975) 159.

10 H.D. Hausen, K. Mertz, E. Veigel und J. Weidlein, Z. Anorg. Allg. Chem., 410 (1974) 156.

11 E.L. Amma und R.E. Rundle, J. Amer. Chem. Soc., 80 (1958) 4141.

12 M. Veith, Z. Naturforsch. B, 33 (1978) 7.

13 M. Veith und O. Recktenwald, Z. Naturforsch. B, 36 (1981) 144.

14 K. Mertz, S. Schwarz, B. Eberwein, J. Weidlein, H. Hess und H.D. Hausen, Z. Anorg. Allg. Chem., 429 (1977) 99.

15 M. Veith, Nachr. Chem. Tech. Lab., 30 (1982) 940.

16 W. Fink, Helv. Chim. Acta, 47 (1964) 498.

17 W.C. Hamilton, Acta Cryst., 12 (1959) 609. 